\section{Participación estudiantil: una mirada crítica, una oportunidad de incidencia política}

\author{
Jazmín Chicas' ${ }^{1}$
}

Palabras claves: democracia, participación activa, género, política pública, Gobierno Estudiantil.

\section{Resumen}

A partir de un estudio de casos de dos centros escolares público-rurales en el municipio de Jicalapa, La Libertad, El Salvador, se explora el fenómeno de la participación estudiantil, encontrando dinámicas que generan instrumentalización y despolitización expresadas en el ciclo de vida de uno de los mecanismos de participación en la escuela, el Gobierno Estudiantil (GE). El enfoque de la investigación busca analizar los factores sociales desde una perspectiva de género que visibilice las desigualdades y afectaciones diferenciadas $e$ identifique las necesidades $e$ intereses particulares de las niñas en el ejercicio de sus derechos en el centro escolar.

Una cultura democrática supone un proceso de construcción social que necesita del diálogo, las relaciones horizontales y la participación para poder concretarse en un escenario real y contribuir así a una sociedad más justa y equitativa. La cultura democrática escolar, entonces, llama a ser parte a toda la comunidad educativa, donde cada persona, desde su posición y condición aportan de forma activa al buen funcionamiento de la escuela y, más ampliamente, al impulso de políticas educativas. En ese sentido, la participación activa y democrática de estudiantes, como parte de la comunidad educativa, más allá de su proceso formativo, invita a pensarles como sujetos y sujetas de derecho, capaces de ofrecer propuestas ante problemáticas que les afectan.

En 1985, el Instituto Interamericano de Derechos Humanos (IIDH) comenzó un

1 Artículo basado en Chicas Marroquín, J. E. (2020). Aproximación a la participación de gobiernos estudiantiles en centros escolares públicos rurales: estudio de casos del municipio de Jicalapa, La Libertad, El Salvador (2008-MAYO 2019) [Tesis de Maestría]. Universidad Centroamericana José Simeón Cañas. 
programa de Educación en Derechos Humanos (EDH), a través del cual se fueron generando estrategias regionales que promovían el desarrollo amplio del derecho a la participación política en el sistema de educación formal. En este mismo informe, se ubica que el cambio de las normas de participación estudiantil se da entre 1990 y los años posteriores; ese fue el punto de partida en el cual los Estados comenzaron a incluir en sus normas educativas alguna forma de organización estudiantil (IIDH, 2007).

Desde un enfoque de derechos humanos, que un Estado legitime y promueva la organización estudiantil es importante y valioso, ya que, primero, abre posibilidades para fortalecer la cultura democrática en el centro escolar, sobre todo para estudiantes, quienes desde la cotidianidad aprenden sobre el derecho a participar, y, segundo, contribuye a la formación y a la acción ciudadana que aporta a la sociedad (IIDH, 2007).

Para el caso de El Salvador, el informe de la IIDH (2007) registra que los Consejos Directivos Escolares y los Consejos de Alumnos eran reconocidos a través de la Ley General de Educación (1996), Arts. 67 y 90; los Lineamientos Generales del Plan Decenal 1995-2005 (1995), y el documento Consejo Directivo Escolar (1996). Una de las formas para visibilizar al gobierno de grado o estudiantil fueron las "Guías metodológicas para la educación en civismo", a partir del año 1996; sin embargo, se detectó un retroceso en la promoción de gobiernos de grado o estudiantiles en planes y programas entre 2000 y 2007 (IIDH, 2007).

Es hasta en el 2005, con el Plan 2021, que se retoma con más fuerza el tema de la participación escolar y se comienza a observar la incorporación del sector estudiantil de manera más clara en los documentos estratégicos del Ministerio de Educación (MINED). Específicamente, en la línea de "Gestión Escolar Efectiva", en el instructivo número 5 de Dirección Escolar Efectiva, establecía estrategias como la conformación del Gobierno Estudiantil (GE) (MINED, 2008b).
Sin embargo, la educación para la ciudadanía en El Salvador, desde la reforma de 1994, ha descansado en una educación en valores éticos, morales y cívicos, lo que desencadenó en la despolitización de la formación ciudadana, ubicando a la persona docente como mero facilitador de "esos valores", obviando la dimensión política de su función (MINED, 2018a).

Teniendo en cuenta esta exploración previa, se planteó que un GE vive un proceso de instrumentalización y despolitización a través de la influencia de factores internos y externos en el centro escolar.

En el primer sentido (instrumentalización), los miembros del GE son incorporados como un comité de festejos o de colaboración para cualquier actividad operativa propuesta por las personas adultas sin acceso a toma de decisiones. Y, en un segundo sentido (despolitización), se considera que no se promueven o se limitan las iniciativas que llevan de fondo una transformación de las relaciones de poder en el centro escolar, o bien la demanda de derechos que, a percepción del estudiantado, están siendo vulnerados.

A su vez, para comprender este fenómeno de la participación estudiantil, será importante detenerse en su naturaleza, de dónde viene y qué la hace tan importante para la cultura democrática, particularmente en la escuela, como un espacio privilegiado para la construcción de nuevas dinámicas de ciudadanía, democracia, convivencia e incidencia política.

Las bases teóricas de referencia han sido estructuradas de forma que acerquen más al fenómeno y al enfoque ontológico desde donde se está intentando abordar. Ubica primero el ámbito de la escuela rural para luego pasar por la participación estudiantil; con estos dos conceptos básicos, toman mayor sentido los conceptos democracia, cultura democrática y ciudadanía, enlazando con la creación de políticas educativas. Todas ellas se complementan desde un enfoque de género para exponer desde el principio una mirada más integral del fenómeno. 


\section{Escuela rural}

Al considerar la reflexión que hace Ortega (citado por Monsalud, 2016), donde expresa que la escuela no nace para el medio rural, sino para un contexto urbano $e$ industrializado, y que justifica su expansión a lo rural por la necesidad de la formación de los futuros obreros, lleva a pensar que en ese esquema hay una invisibilización de las propias características de la escuela rural, las cuales pueden ser en el ámbito de la participación.

Otra postura interesante es la de Vásquez Recio (2016), quien introduce la reflexión sobre la expansión de la escuela rural como una garantía de bien común. En su papel socializador, la escuela rural viene a ser el medio por el cual se deberían equiparar las condiciones para la población más alejada de la zona urbana, garantizando así la igualdad de condiciones y oportunidades para el estudiantado, así mismo su construcción de ciudadanía que le permita tener una vida digna, una proyección laboral y una estabilidad económica.

La autora también plantea la necesidad de reconocer la diversidad de la escuela rural y abandonar la idea de querer encajonarla en el sistema educativo urbano, y para ello expone la situación de la escuela rural desde el sistema de desigualdad y el sistema de exclusión ocupados por las sociedades capitalistas, explicando que la escuela rural se mueve entre ambos sistemas. Al intentar someter a las escuelas rurales a prototipos creados para escuelas urbanas, se invisibiliza la riqueza que puede aportar al sistema educativo $y$, en efecto, no responde a sus propias necesidades, lo que las deja en desigualdad social y educativa.

\section{Participación en el sistema educativo}

El sistema educativo es la estructura que da vida a la educación como un derecho, tanto en el ámbito formal como en el inclusivo comunitario, garantizando o debiendo garan- tizar la educación a lo largo de la vida. Santos plantea que desde las estrategias que dicho sistema pueda impulsar se puede fortalecer o atrofiar la participación de todos los actores involucrados, particular y especialmente la de los y las estudiantes:

"De hecho, con la misma legislación, con las mismas estructuras, con el mismo currículum básico, podemos encontrar centros que la cultivan y otros que la recortan o destruyen" (Santos Guerra, 2003).

Sarramona i López y Rodríguez Neira (2010) plantean la importancia de la participación en el sistema educativo a partir del fin principal de la educación para transformar las sociedades, exige diálogo, negociación, diferencias o disidencias, llevando a tomar decisiones a partir de escuchar las necesidades de todas las partes involucradas, en este caso la comunidad educativa.

La participación debe ser parte del proyecto educativo, respetando las funciones, responsabilidades, deberes y derechos de cada una de las personas que son parte de la comunidad educativa. En otras palabras, es la clave para concretizar un proyecto educativo basado en una cultura democrática que prepara a sus integrantes para ejercer su ciudadanía, particularmente a estudiantes.

\section{Participación estudiantil}

La escuela, al ser un escenario privilegiado para ejercer ciudadanía y aprender a vivir en una cultura democrática, debe proporcionar a los y las estudiantes experiencias significativas y reales de participación activa.

Para Sarramona i López y Rodríguez Neira (2010), la participación estudiantil plantea tres finalidades educativas. La primera tiene que ver con educar en los principios democráticos; la segunda es establecer un currículo que permita la autoformación, y la tercera, desarrollar el espíritu crítico. Estas se deben adaptar a las características y necesidades en 
cada centro educativo y a nivel de las decisiones macropolíticas en el sistema educativo.

En el caso del MINED (2008), a través del Manual de Participación Estudiantil para el Fortalecimiento de la Convivencia Escolar, define participación estudiantil como el conjunto de actitudes y acciones individuales, colectivas y organizacionales de los estudiantes como miembros de la comunidad educativa.

\section{Participación política}

La participación desde el enfoque de derechos obliga a ver a la persona como sujeta de derechos, sin distinciones por edad, sexo, religión u otras variantes. Una participación activa lleva a la persona o al colectivo a identificar necesidades y demandas en su entorno social, buscando mecanismos que lleven a soluciones concretas. La participación activa de los y las estudiantes conlleva a la organización y demanda de la garantía de sus derechos, lo que la vuelve una participación política que busca el diálogo, la negociación y la incidencia en la mejora de la vida escolar.

El concepto de participación política que presenta Sabucedo (1988) habla de dos tipos de participación política, la convencional y no convencional. La primera relacionada con procesos electorales y en la segunda hace alusión a gestionar peticiones, manifestaciones legales. Ambas formas de participación política pueden diferenciarse atendiendo al criterio de demanda o no de las mismas por parte del sistema.

Así como la participación política convencional es fomentada y animada desde las instancias del poder constituido, con lo que puede ser fácilmente controlada y canalizada, la participación política no convencional, en ocasiones, desborda los mecanismos instituidos de participación y supone un enfrentamiento con la legalidad establecida, aunque este enfrentamiento no es por naturaleza violento.
Y, en esa misma línea, la participación de las mujeres se vuelve un acto político; más allá de una paridad en número, se pone sobre la mesa cómo las relaciones de poder entre los géneros obligan a las mujeres, en este caso niñas y adolescentes, a adoptar ciertos roles o no en la dinámica del GE.

La autora María Seca invita a hacer una reflexión interesante sobre cómo superar las visiones de participación androcéntricas y adultocentristas que no permiten una participación real, sobre todo para las mujeres jóvenes. La construcción de lo "juvenil-masculino" como universal que "legitima una relación de dominación inscribiéndola en una naturaleza biológica que es en sí misma una construcción social naturalizada" (Bourdieu, citado por Seca, 2016), al mismo tiempo que estabiliza y refuerza las diferencias de género, concretizándolas en la vida cotidiana (Seca, 2016)

Por tanto, la intención es enmarcar la participación como un derecho que permite a la persona o al colectivo el pleno goce de los mismos y, en esa misma condición, ser partícipe de su propia construcción como ciudadano y ciudadana, no para el futuro sino desde el presente, concretizándolo en la cotidianidad de la dinámica escolar y en las políticas educativas que lo promueven.

\section{Democracia y género}

No es de interés profundizar en las raíces de la democracia, pero sí exponer una propuesta diferente para tomarla como referencia. La referencia histórica de la democracia, como ya se sabe, está en la antigua Grecia, y en su nacimiento ya se desvela una desigualdad al interpretar que el ser ciudadano partícipe de la democracia no incluía a las mujeres.

Cobo Bedía (2008) define la democracia como el modelo de organización social y política que defiende los mismos derechos formales para todos los individuos, basándose en la igualdad ante la ley y en la imparcialidad de la misma; a su base está la univer- 
salidad, la cual abre camino a la igualdad al partir de la idea de la razón que caracteriza al individuo y la cual es capaz de liberarlo; sin embargo, la autora expone que el problema se encuentra en el momento de concretizar dicho valor universal, donde para las mujeres ya no es tan fácil, basándose en diferencias "naturales" con la intención de hacer una diferenciación en cuanto a la posición desigual de hombres y mujeres en una sociedad democrática, ejemplo de ello son las expresiones de Kant o de Rousseau con el contrato social.

Una propuesta para romper con el contrato social de Rousseau se encuentra en comprender la democracia desde dos perspectivas que, al unirlas, configurarían una base para comenzar a hablar de ciudadanía activa.

La primera: democracia participativa. Para Romero (2005), la democracia participativa es una expresión de una nueva forma de democracia directa que amplía los espacios de representación y participación de la ciudadanía, dándole un rol más protagónico en el proceso deliberativo. Es la ciudadanía la que, a través de mecanismos participativos e inclusivos, delibera y decide sobre los asuntos que afectan su calidad de vida, revalorizando y resignificando el ejercicio de ciudadanía.

La segunda: no es posible ampliar la democracia y la ciudadanía para las mujeres si estas no se construyen como un actor social con capacidad de intervención social y de negociación política, lo que lleva a la necesidad de exponer otro concepto clave, la democracia paritaria, la cual es planteada por Amorós (2008, citada por Cobo Bedía, 2008) en el mismo artículo, como un instrumento para la vindicación del contrato social, afirmación que parte del supuesto que el contrato social, aunque fue basado en la universalidad de los derechos, no se concretiza de la misma manera para las mujeres por no considerarlas como sujeta política.

Por tanto, se habla de una democracia que debe visibilizar tanto a hombres como a mujeres, donde esa universalidad de derechos políticos y civiles se concreticen en tomas de decisiones sin distinción ni discriminación por sexo ni por edad.

\section{Ciudadanía y género}

El Instituto Nacional de las Mujeres en México construye una definición que resulta relevante para la investigación, definiendo ciudadanía como el conjunto de derechos y deberes que hacen de cada persona un miembro de la comunidad política. Establece la calidad del vínculo entre Estado y persona; a través de esa relación, se establecen prerrogativas para participar en la vida pública y en la toma de decisiones de una comunidad o de una nación. Por lo tanto, la ciudadanía no implica solo derechos y responsabilidades, sino también capacidad de participación (Ugalde y Larralde, 2008).

La relevancia en poner a discusión la ciudadanía desde el enfoque de género es que para la investigación es de importancia situar a la sujeta y al sujeto de derecho, el o la estudiante, como una persona que se está preparando para ejercer ciudadanía $y$, por ello, deberán buscarse las estrategias necesarias para que sea en igualdad de condiciones para hombres y mujeres, aprovechando el espacio formativo que representa el participar en el GE para tal fin.

\section{El ciclo de las políticas públicas}

Para Ordóñez-Matamoros (2013, p. 30), la política pública es "el conjunto de acciones implementadas en el marco de planes y programas gubernamentales diseñados por ejercicios analíticos de algún grado de formalidad, donde el conocimiento, aunado a la voluntad política y los recursos disponibles, viabilizan el logro de objetivos sociales".

Los objetivos sociales incluyen el ámbito de la educación y parece relevante compartir esta perspectiva porque resalta el conocimiento y el conjunto de decisiones por actores claves en todo el ciclo del diseño de la política. 
Es de vital importancia que para que la política pública formulada logre los objetivos para los que fue creada cuente con parámetros claros que le permitan un seguimiento, identificando avances, obstáculos y oportunidades, y así hacer los cambios necesarios en una realidad que también es dinámica.

También es necesario que la población objeto de la política perciba el problema como tal y sea parte de la propuesta de las soluciones, de modo que se construya un ambiente favorable para el desarrollo de la política desde la participación democrática (Ordóñez-Matamoros, 2013).

Para el monitoreo y la evaluación dentro del ciclo de las políticas públicas, deben considerarse ejercicios constructivos y de aprendizaje, tanto de los aciertos como de los desaciertos, de modo que toda la información generada de la experiencia se pueda gestionar como conocimiento, previendo problemas futuros (Ordóñez-Matamoros, 2013).

El diseño de una política pública también marca la forma en que será implementada. El nivel de sentido de pertenencia que le dan los $y$ las actoras en el territorio es clave para ello en un contexto determinado. Resulta importante un análisis integral desde la macro y la micropolítica acorde a lo anterior, que permita identificar las interrelaciones, ideologías, significados socioculturales y relaciones de poder que permiten el éxito de una política pública.

\section{Macro y micropolítica}

Desde el ámbito educativo, es de interés plantear cómo se entenderán la macropolítica y la micropolítica, como dos ámbitos que se interrelacionan e influyen en el camino de los sistemas educativos en un país.

Desde la visión de Bardisa Ruiz (1997), se define la macropolítica como "la forma en que los grupos de interés del entorno tratan de imponer sus lógicas de acción en la organización"; en este caso, la organización sería la escuela y las lógicas se pueden traducir en la legislación, las políticas educativas, los manuales e instructivos emanados desde la institución que rige el sistema educativo y el papel de los actores que inciden en su desarrollo desde los entornos ya detallados.

Esta visión se complementa con la micropolítica, la cual tiene a su base, según Ball (citado por Bardisa Ruiz, 1997), las relaciones de poder, los conflictos y las negociaciones que se dan dentro de la organización, es decir, dentro de la escuela. En el mismo artículo, Santos Guerra (2003) plantea que ambas visiones son necesarias para poder entender la organización escolar en toda su dimensión.

Identificar ambos ámbitos (macro y micro) y cómo se interrelacionan en torno a la participación estudiantil a través del GE resulta importante como clave de análisis.

\section{Marco normativo de El Salvador a favor de la participación de la niñez y la adolescencia}

En su marco normativo, El Salvador cuenta con algunas herramientas para promover la participación de la niñez y la adolescencia.

La primera es la Ley General de Educación, por medio del artículo 3, literal h y el artículo 90 "Derechos de los educandos", literal f; la segunda es la Ley de Protección Integral de la Niñez y Adolescencia (LEPINA), en su título IV "Derecho a la participación", y una tercera es la Política Nacional para la Convivencia Escolar y Cultura de Paz, la cual, entre sus ejes y estrategias, promueve la participación y el empoderamiento de niñas, niños y adolescentes (NNA) como sujetos y sujetas de derecho. En el 2005, con el Plan Nacional de Educación 2021, específicamente, en la línea de Gestión Escolar Efectiva, en el instructivo número 5 de Dirección Escolar Efectiva, se establecen estrategias como la conformación del GE (MINED, 2008b).

Si bien este marco normativo muestra avances con respecto al derecho a la participación, posiblemente no logra ser suficiente para contribuir a un cambio en el imaginario 
social sobre el rol del estudiantado y de la planta docente con respecto al ejercicio de la ciudadanía.

A más de diez años de venir implementando estrategias para la participación estudiantil, a través de políticas educativas y la implementación de programas vinculados a la convivencia escolar, resulta relevante conocer la experiencia de participación estudiantil en centros escolares públicos de la zona rural, dado que frecuentemente se percibe que carecen de iniciativas de innovación y organización.

Es entonces cuando resulta interesante ver la experiencia del municipio de Jicalapa, en el departamento de La Libertad, con dos centros escolares públicos del área rural que han sido parte de una vivencia de participación estudiantil a través de la implementación de GE y con la participación de diversos actores, en el marco temporal de 2008 a mayo de 2019, por ser un periodo particular del impulso de estrategias de participación estudiantil desde un enfoque de derecho.

Tomando en cuenta que no hay estudios previos sobre esta estructura en el centro escolar en el país y que se busca indagar desde la propia experiencia de los y las estudiantes principalmente, se optó por una investigación de tipo cualitativo, partiendo de un diseño metodológico de estudio de casos en su visión de estrategia metodológica.

Con una intención de doble triangulación la cual se realizó a partir de distintas técnicas (entrevista, grupo focal y análisis de contenido) y diversos sujetos y sujetas claves que interactúan en la problemática estudiada (docentes, estudiantes, representantes del MINEDUCYT y representantes de organizaciones de sociedad civil), en total, se realizaron 12 entrevistas, 2 grupos focales y el análisis de contenido a tres instrumentos/ lineamientos del MINEDUCYT orientados a la participación estudiantil en el periodo de 2008 a mayo de 2019.

Para dialogar sobre el tema, la reflexión se centrará en dos líneas: la primera sobre la desarticulación entre política educativa a través de instrumentos/lineamientos que se desprenden de ella y la implementación del GE en el centro escolar, y la segunda sobre los factores externos e internos que influyen en la participación del estudiantado a través del GE y que contribuyen a su despolitización $e$ instrumentalización, debilitando su potencialidad de incidencia en favor de los derechos del estudiantado.

\section{Desarticulación entre política educativa e implementación del GE en el centro escolar}

Las políticas educativas se han considerado el medio por el que un Estado expresa sus obligaciones, concretizándolas a través de acciones estratégicas para lograr avances en la garantía de los derechos. En El Salvador, los pocos mecanismos de participación estudiantil se enmarcan dentro de políticas educativas vinculadas a la convivencia escolar. A falta de una política educativa particular que garantice la participación estudiantil, la investigadora identificó tres instrumentos/lineamientos que abordan estrategias para la participación del estudiantado.

Estos son el Manual de Participación Estudiantil para el Fortalecimiento de la Convivencia Escolar, del MINEDUCYT; la Guía para implementar el Gobierno Escolar en los centros educativos, y la Caja de herramientas para docentes y educadores "Ciudadanía, Memoria y Cultura de Paz en El Salvador. Convivencia y cultura de paz" (Vol. 3). A través de un análisis de contenido, se irán encontrando elementos que dificultan la articulación entre la normativa y su implementación en los centros escolares.

Una normativa clara permite establecer estrategias que respondan a las necesidades por las que fue creada y construyan puentes de diálogos y construcciones colectivas. Los instrumentos/lineamientos en cuestión, si bien establecen algunas orientaciones, estas no son muy claras y están desarticuladas con la implementación en el centro escolar, tanto a 
nivel estructural, argumental y de aplicación en el centro escolar.

Para lograr una implementación satisfactoria de las políticas educativas, deben alinearse diferentes elementos, uno de ellos es que los programas y las normativas que se deriven de ellas garanticen de alguna manera la participación activa de las personas sujetas de derecho en todo su ciclo de implementación. De este modo, las sujetas de derecho reconocerán el fin de la creación e implementación de dichos programas y lineamientos, en relación con este punto; el estudio de caso identificó que ese reconocimiento es deficiente o inexistente. La forma de rescatar estos instrumentos/lineamientos es, entonces, a través de los manuales que desarrollan las organizaciones de sociedad civil (OSC), ya que para elaborarlos retoman los documentos formales del MINEDUCYT, agregando sus propias particularidades al proceso.

En el caso de docentes y direcciones, estos reconocen el manual que cada institución lleva al centro escolar, por ejemplo, el que implementa el Tribunal Supremo Electoral o el manual que llevan las OSC, que se encuentra implementado como proyecto en el centro escolar. En el caso de estudiantes, la situación todavía es más compleja, ya que reconocen solo las orientaciones que sus personas referentes adultas les dan.

En la misma línea, al no definir lineamientos claros $e$ inclusivos, se abren oportunidades para que en la conformación se mezclen valores impuestos por las personas adultas que dirigen el proceso. Por ejemplo, el perfil de estudiantes que pueden participar en el GE o quiénes conforman el tribunal electoral. Estos valores, según lo encontrado en la investigación, excluyen a estudiantes por edad, rendimiento académico, grado académico y prejuicios en relación con quién es líder o no, como lo expresa un estudiante entrevistado: "Sacaron a los alumnos que tenían buena imagen, buenas notas, los profesores, y ese grupo es el que iba a concursar para presidente".
Otro elemento ausente en la experiencia estudiada y de mucha importancia para reflexionar es la dificultad para identificar insumos claros en relación con el seguimiento y la evaluación de la estrategia de participación del estudiantado a través del GE. Lo que actualmente se registra se hace solo a nivel cuantitativo, privando de un análisis integral que pueda brindar elementos cualitativos, generando insumos para la toma de decisión y la mejora en la implementación del mecanismo de participación.

Lo anterior se vuelve difícil cuando hay una percepción en direcciones, docentes y estudiantes, quienes relacionan el GE con una estrategia promovida desde las organizaciones externas que lo impulsan, limitando la apropiación del proceso y su propia sostenibilidad; en este punto se profundizará un poco más en la segunda línea de reflexión.

Esta percepción de ausencia del MINEDUCYT, en el caso particular del GE, podría deberse a la misma estructura de cómo se le da seguimiento al mecanismo de participación estudiantil.

A nivel central y departamental, el área identificada como la instancia responsable del seguimiento a los procesos de participación de los GE fue el Departamento de Arte, Cultura, Recreación, Deporte y Ciudadanía. Sin embargo, también se cruza con el área de asesoría pedagógica, ya que el GE debe participar en la planificación estratégica escolar. Esta inconsistencia en la estructura interna genera confusión en el centro escolar, y se acude con mayor facilidad a la persona referente de las OSC, antes que al MINEDUCYT, para este proceso en particular.

Así mismo, se identificó como una limitante la carencia de un enfoque geográfico que considere las características particulares de la zona rural, desaprovechando la valiosa oportunidad de incorporar las capacidades culturales, sociales y organizativas que puedan abonar al proceso de conformación. Esta carencia se traduce en la práctica en procesos homogeneizados donde con características 
diferentes de cada contexto se pretenden iguales resultados. Todo lo anterior, sin una transversalización del enfoque de género de forma clara y concreta, tanto a nivel de lineamientos como en la práctica en el centro escolar, con diferentes matices y avances.

Si bien se puede apuntar un avance en el lenguaje inclusivo, en el reconocimiento de la necesidad de la participación en igualdad de condiciones, si se queda solo a nivel de discurso y no se buscan transformar las prácticas cotidianas, se corre el riesgo de seguir perpetuando prácticas que limitan una participación activa en igualdad de condiciones para niños y niñas en el GE.

Un enfoque que debe estar presente en todo el ciclo de vida del GE, ya que cada una de las fases da la oportunidad para hacerlo. En la conformación del GE, promoviendo una participación equitativa y en igualdad de condiciones, libre de prejuicios por ser niña o niño. En el caso de la investigación, no se tuvo acceso a datos estadísticos para dar una mirada a la conformación de los GE, en tanto niñas y niños. De la misma forma, en las entrevistas con actores claves, se identificaron elementos tímidos sobre las dificultades de las niñas a la hora de participar, como por ejemplo no poderse reunir en horario contrario al de su jornada escolar por cumplir con tareas del hogar.

En la implementación, al momento de la creación del plan de trabajo del GE. Sin embargo, se identificó que se prioriza un enfoque de representatividad, dificultando identificar con claridad el involucramiento del estudiantado más allá del ejercicio de votación, acompañado de un discurso de igualdad, pero que no se percibe que trascienda a la practicidad en la cotidianidad. Contribuye así a la elaboración de un plan de trabajo con actividades generales, sin mecanismos de consulta que promuevan una participación más activa, que expresen las necesidades $e$ intereses de niñas y niños $y$, por ende, un GE que responda a las mismas.
Se trata entonces de hacer perceptible la invisibilización que muestren los estereotipos de género en la cotidianidad frente a los discursos de igualdad. Por ejemplo, cuando una niña menciona que al momento de hacer actividades ellas recogen la basura y los hombres hacen lo más pesado o cuando un docente afirma que las niñas por ser niñas son más responsables y por eso están en el GE.

\section{Factores externos e internos que influyen en la participación del estudiantado a través del GE}

La segunda línea de reflexión busca profundizar en los factores externos e internos que influyen en las características que toma la participación del estudiantado a través del GE; si bien guarda relación con los elementos antes expuestos, se hace énfasis en las formas de funcionar del GE en todas sus fases.

Las características de instrumentalización y despolitización se hacen presentes a través de prácticas que van desde quiénes pueden o no participar en él, cómo se eligen las funciones dentro de la estructura, la toma de decisiones frente al trabajo a realizar y la sostenibilidad del mecanismo de participación.

Un primer factor es la asignación de las funciones. El estudio de caso permitió identificar que estas son definidas en una reunión dirigida por las direcciones, los docentes y el personal técnico de las OSC que implementaba el proyecto, exceptuando la función de presidencia y vicepresidencia, que sale de las elecciones del estudiantado. Estas funciones asignadas nuevamente se ven cargadas por estereotipos de edad, rendimiento académico y percepción de "buen estudiante", como lo menciona un docente en la entrevista: "Vamos viendo lo idóneo, lo proponen los mismos alumnos o nosotros". Hasta el momento, no hay forma de identificar por sexo quiénes han asumido estas funciones, elemento que podría haber sumado más al análisis de género en el tiempo.

El segundo factor es la toma de decisiones. Este factor puede llegar a determinar el grado 
de autonomía por parte de las personas sujetas de derecho en un espacio.

Aparentemente, en la investigación realizada, no se identifica una preferencia en la toma de decisiones por distinción de sexo, sino más bien una con base en jerarquía; en este caso, la presidencia es la que posee una carga de poder más claramente delegada y es la que se relaciona con la dirección y con el referente de la OSC, posteriormente ella delega para que "los demás integrantes del GE hagan". Esto podría contribuir a una participación menos activa de parte de los demás integrantes del GE, como se puede apreciar en la figura 1 .

Figura 1. Interpretación de la toma de decisiones en el GE desde la perspectiva estudiantil

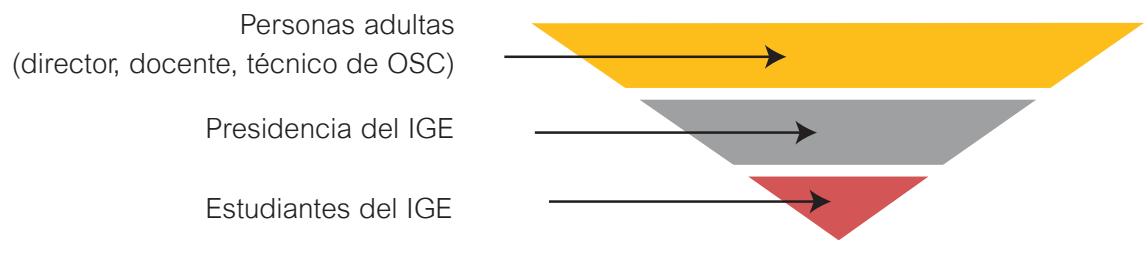

Fuente: elaboración propia, a partir de lo recabado en las entrevistas con estudiantes para la investigación.

En la misma línea, los y las estudiantes identifican que dependen en gran medida del permiso que den docentes y dirección para la implementación de su plan de trabajo. La presencia de las personas adultas para la toma de decisión del GE es indispensable, indicando expresiones adultocéntricas, estableciendo una diferencia entre niñez y adultez, que denota el poder de la persona adulta y se limita la participación del niño o niña por no considerarla una persona apta para desenvolverse sin la tutela adulta (Duarte Quapper, 2012).

$\mathrm{Y}$, finalmente, un tercer factor es la autonomía del GE, analizada desde tres elementos claves: reconocimiento, gestión y sostenibilidad.

Primero, el reconocimiento. El GE goza hasta el momento de un reconocimiento formal, a través de los lineamientos de parte del MINEDUCYT, lo que da fuerza y vigencia a su existencia y la exigibilidad de la misma. A nivel informal, en el centro escolar, su reconocimiento se ve ligado al tipo o tipos de beneficios que la escuela logré a través del mismo. Mejora en infraestructura, fachada, mejora del refri- gerio al gestionar otras alternativas, mejora de espacios recreativos como canchas o bancas.

Si en cambio el GE comenzara a proponer cambios más significativos, estructurales en relación con demandas de derechos vulnerados, el reconocimiento se vería amenazado. Como lo expresa un director en una entrevista "Al principio, no estaban muy de acuerdo porque no confiaban en los jóvenes", al comentar cómo la planta docente percibe al GE.

A raíz de lo expuesto sobre el reconocimiento, se comienzan a identificar elementos que dan pauta de una instrumentalización en la participación de los y las estudiantes. Son usuarias en la dinámica de participación en la escuela, con información sobre lo que se realizará, pero sin control del cómo y cuándo realizarlo, manteniéndose en el margen de lo permitido por las personas adultas para que su trabajo sea valorado como "bien visto", sin trascender a cambios significativos en el proyecto educativo del centro escolar.

Segundo, la gestión. Los GE dependen del apoyo y voluntad de las autoridades de la escuela, específicamente de la dirección o 
de las OSC que en ese momento estén implementando un proyecto en la escuela y que involucre a esta estructura.

Es decir, los GE sí están enterados de las gestiones, redactan sus cartas, identifican con quienes pueden generar alianzas, pero no lo hacen por sí solos. "Hubo veces que le pedíamos al alcalde para poder arreglar la cocina a través de una carta", expresó una niña en un grupo focal. Sin embargo, al conversar con representantes de la municipalidad, esta institución no reconocía al GE como estructura con quien dialogaba, sino que al director o al referente de las OSC porque, posiblemente, en estas figuras veía el liderazgo y la formalidad de la petición.

De igual manera, la gestión interna para las reuniones necesarias para el trabajo del GE eran permitidas en la medida en que una persona adulta lo solicitara. Por ejemplo, para reunirse y planificar las actividades, como se ha mencionado en otro apartado, los y las estudiantes no recuerdan haberse reunido alguna vez solos como GE, sino siempre con la presencia de docentes, dirección o técnicos de las OSC.
Tercero, la sostenibilidad. Esta se ha relacionado con las posibilidades de la continuidad de la estructura y con el sentido de pertenencia hacia la estructura. Darder Vidal (2008) asocia la participación con el sentido de pertenencia al expresar: "Sentirse parte es asumir la necesidad de intercambiar, escuchar y dialogar con los demás y examinar cada situación para contrastar con las necesidades y las expectativas de la sociedad".

En cuanto a las posibilidades de continuidad, por lineamiento del MINEDUCYT en el manual de participación estudiantil, el GE tiene una vigencia de dos años. En el centro escolar, aunque se tiene claridad del tiempo de legislatura del GE, según el estudio realizado, la continuidad, más que de ese periodo formal, depende en gran medida de la voluntad de las direcciones y de la presencia de un proyecto por parte de una OSC. "No sé, no nos han dicho nada", así lo expresa una niña en un grupo focal al consultarles cómo pensaban continuar con el trabajo del GE para el 2020.

De forma resumida, se puede apreciar todo este apartado desde la figura 2.

Figura 2. Resumen de los factores internos y externos que influyen en la implementación del GE

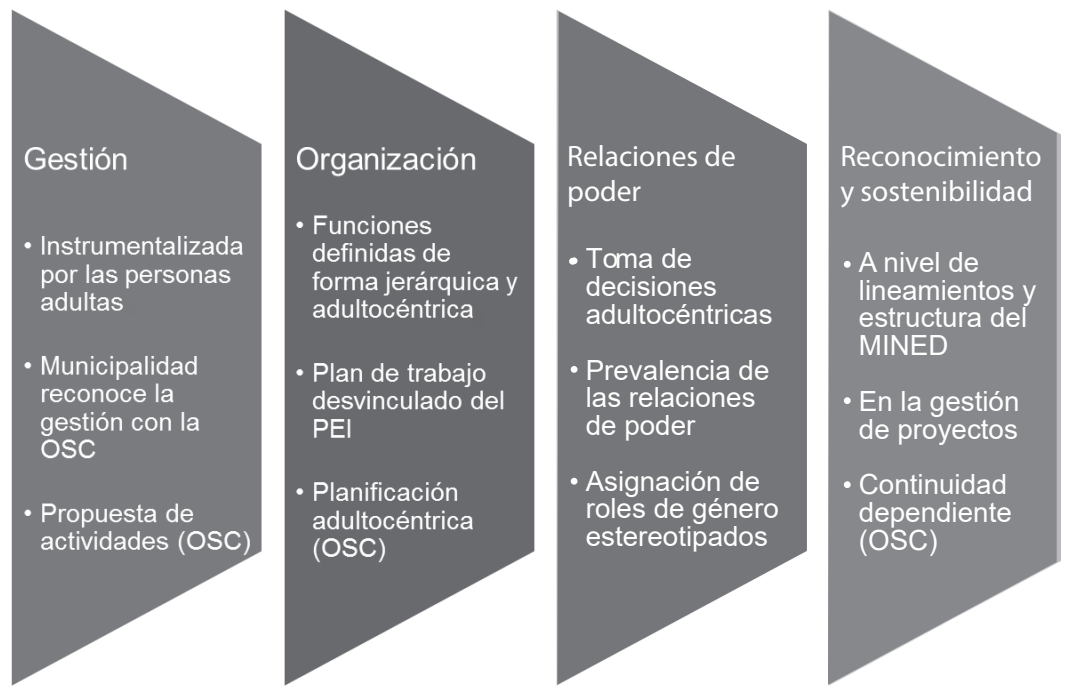

Fuente: elaboración propia, a partir de las entrevistas realizadas para la investigación. 
Por todo lo anterior y teniendo en cuenta la adaptación metodológica realizada para la investigación, con base en la propuesta de Hart (1993) y Murguialday y Vásquez (2005), se define como:

- Participación pasiva: los y las estudiantes son usuarias en la dinámica de participar en la escuela, sin información, sin control y sin toma de decisión.

- Participación obediente: los y las estudiantes son usuarias en la dinámica de participación en la escuela con información, pero sin control ni toma de decisión.

Las dos categorías anteriores corresponden a una participación despolitizada $e$ instrumentalizada.

- Participación simulada: los y las estudiantes son consultadas en la dinámica escolar, expresando sus necesidades $e$ intereses, pero no son tomadas en cuenta a la hora de la toma de decisiones.

- Participación activa: los y las estudiantes se organizan y deciden de forma autónoma en las iniciativas para resolver sus necesidades e intereses.

Estos dos niveles son identificados como participación política autónoma.

Para el análisis de la participación estudiantil a través de los dos GE de los centros escolares públicos rurales que participaron en el estudio de caso, se considera lo siguiente:

En uno de los centros escolares, el GE se acerca a una "participación simulada". Según lo definido para la investigación, esto implica que los y las estudiantes son consultadas en la dinámica escolar, expresando sus necesidades $e$ intereses, pero no son tomados en cuenta a la hora de la toma de decisiones.

Lo anterior se hace presente al identificar que hay un nivel de consulta con la estructura, pues mantiene su continuidad después de la implementación del proyecto de parte de la OSC, aunque con menor actividad.

Sin embargo, se observan algunos elementos en contra, como el que aún no es tomado en cuenta en la toma de decisiones como parte del Plan Estratégico Institucional (PEI) y en el Plan Escolar Anual (PEA); por el contrario, su continuidad depende de la voluntad de las autoridades de la escuela y el tipo de actividades realizadas en su mayoría son dirigidas por las personas adultas, como el huerto escolar y la gestión para el arreglo de la cocina, entre otras.

En el caso del otro centro escolar, este responde a una "participación obediente". Según lo definido para la investigación, ello implica que los y las estudiantes son usuarias en la dinámica de participación en la escuela con información, pero sin control ni toma de decisión.

Esta situación es observable al identificar que se les informa sobre el proyecto a implementar, son parte de él en su periodo de implementación, pero sin control ni toma de decisión; no se les toma en cuenta en la construcción del PEI y en el PEA del centro educativo, y, terminado el proyecto, no se les explica ni se les informa que la estructura ya no continúa activa y tampoco los y las estudiantes se pronuncian por el funcionamiento de la misma.

A pesar de que los centros escolares del estudio se encuentran en diferente nivel de participación, uno en obediente y otro en simulada, ambos presentan características de instrumentalización y despolitización.

Al respecto, Carbajal (2013) expresa que, con respecto a las estructuras de participación estudiantil como oportunidad para un diálogo plural, lo que se ha observado es que "en los hechos han predominado los enfoques paternalistas, donde las voces de los estudiantes tienden a ser acalladas o relegadas". La inclusión en la participación ciudadana en el centro escolar implica que el estudiantado tenga un verdadero rol como tomadores y 
tomadoras de decisión en los asuntos que afecten sus vidas; esto es parte de la participación política.

Sin embargo, los elementos observados en la investigación denotan que hay una intención de tomarles en cuenta, pero no en las mismas condiciones, lo que resulta ineficaz y nominal para la construcción de la ciudadanía activa.

Al considerar el sistema educativo como eso, un sistema, es importante señalar la importancia que cada uno de los actores implicados en ese sistema representan para ser parte en la mejora de la calidad del mismo; de esa manera, se vuelve un ganar-ganar personal y colectivo.

Exige, entonces, reconocer que un GE tiene dos potencialidades importantes: la educativa y la democrática. Como una estructura con fuerza colectiva en su potencial educativo, genera oportunidad de aprendizaje en el estudiantado, desde la práctica y la cotidianidad aprendiendo a participar de forma activa, integrándose en la estructura del centro educativo en la toma de decisiones ante las situaciones que les afectan como estudiantes, lo que lleva al elemento potencial de la democracia.

A la luz de los resultados expuestos, será importante retomar, primero, la urgente necesidad de elevar a nivel de política educativa la participación estudiantil y una de sus formas debe ser el GE, aunque hay otras. Si bien los instrumentos son un avance, estos no son suficientes para garantizar en tanto enfoques y herramientas para hacer vida la participación en la escuela como parte de la gobernanza escolar.

Luego, el monitoreo constante y presente juega un papel clave respecto a generar el conocimiento basado en evidencia para que pueda generar las estrategias que fortalezcan la acción transformadora y democratizadora del sistema educativo MINEDUCYT. En el caso del GE, requiere la recolección de información sistemática que dé cuenta del trabajo de los GE tanto a nivel estadístico como en sus procesos de desarrollo.

El monitoreo se complementa con la evaluación. Si bien hay un lineamiento que la menciona, esta no es exclusiva para el GE, sino de la participación estudiantil en general y enfocada hacia la convivencia escolar. No obstante, es clave generar los espacios de evaluación con enfoque de participación activa que contribuyan a retroalimentar $y$ mejorar el proceso, sabiendo que las políticas educativas per se no garantizan su implementación en el territorio y que la ciudadanía no implica solo derechos y responsabilidades, sino también capacidad de participación (Ugalde y Larralde, 2008).

Es importante considerar cómo se puede fortalecer la articulación entre MINEDUCYT y las OSC u otras instituciones externas que intervengan en la dinámica escolar, ya que, a falta de esa articulación, los procesos del GE se pueden volver muy dependientes de la presencia o ausencia de estos actores en la escuela.

Otra consideración importante es que la escuela rural presenta un gran reto para el sistema educativo. Es importante que se formulen políticas públicas pensando en combinar las características de la zona (contexto, oportunidades, cultura organizacional), las y los actores presentes en el territorio y las múltiples fuerzas político-institucionales para lograr políticas apegadas a la realidad y necesidades de la población sujeta de derechos.

Esto requiere, entonces, romper paradigmas generalistas sobre política educativa, donde se da una respuesta a problemáticas sociales que son vivenciadas desde diferentes realidades. Coincidiendo con el planteamiento de Vásquez Recio (2016), en la necesidad de reconocer la diversidad de la escuela rural y abandonar la idea de querer encajonarla en el sistema educativo urbano.

Para superar las características de instrumentalización y despolitización en la participación estudiantil a través del GE, se debe 
de apelar al principio de la educación para transformar las sociedades. Carbajal (2013) expresa que "la calidad de la educación no sólo implica el desarrollo de conocimientos y habilidades cognitivas, sino que una educación de calidad incluye también el desarrollo de habilidades para una ciudadanía responsable dentro de ambientes emocionalmente enriquecedores". Y parte de la democratización de la calidad educativa es brindar al estudiantado oportunidades de participación reales, en términos equitativos, entre personas adultas, la niñez y la adolescencia como sujetas y sujetos de derecho.

Para cerrar, aunque un estudio de casos no se puede generalizar, sí puede afirmarse sobre la base de los resultados que aporta pautas concretas de lo que está sucediendo con respecto a un fenómeno para comprenderlo y motivar una investigación más amplia en esta línea.

El centro escolar sigue siendo un lugar privilegiado en donde se transmiten y reproducen valores, actitudes y comportamientos, naturalizados en nuestra sociedad, pero al mismo tiempo constituye un factor de cambio para transformar realidades y fomentar la aplicación de derechos y libertades. Y sola no puede con esta tarea. Si se quiere una educación de calidad, que implica elementos como democracia, ética, justicia e inclusión, será necesario el involucramiento de toda la comunidad y de toda la sociedad de forma articulada, actuando como sistema.

\section{Referencias bibliográficas}

Bardisa Ruiz, T. (1997). Teoría y práctica de la micropolítica en las organizaciones escolares. Revista Iberoamericana de Educación, 15, 13-52. https://rieoei.org/historico/oeivirt/ rie15a01.pdf

Cobo Bedía, R. (Ed.). (2008). Repensando la democracia: mujeres y ciudadanía. En Educar en la ciudadanía: perspectivas feministas (pp. 19-52). Los Libros de la Catarata.
Carbajal, P. (2013). Convivencia democrática en las escuelas. Apuntes para una reconceptualización. Revista Iberoamericana de Evaluación Educativa, 6(2), 13-35. http://www. rinace.net/riee/numeros/vol6- num2/art01.pdf

Darder Vidal, P. (2008). Las dimensiones personales, base de la participación. En J. Gairín Sallán y S. Antúnez Marcos (Coords.), Organizaciones educativas al servicio de la sociedad (pp. 478-483). Wolters Kluwer.

Duarte Quapper, C. (2012). Sociedades adultocéntricas: sobre sus orígenes y reproducción. Última Década, 20(36), 99-125. https:// www.scielo.cl/pdf/udecada/v20n36/art05.pdf

Hart, R. (1992). La participación de los niños. De la participación simbólica a la participación auténtica. Ensayos Innocenti, 4, 1-46. UNICEF. https://www.unicef-irc.org/ publications/pdf/ie_participation_spa.pdf

Instituto Interamericano de Derechos Humanos (IIDH). (2007). VI Informe Interamericano de la Educación en Derechos Humanos. https://www.iidh.ed.cr/multic/ UserFiles/Biblioteca/IIDH/3 2010/484ee56ac4a5-4c7b-a29b-62f9ae2e651a.pdf

Ministerio de Educación [MINED]. (2008a). Manual de Participación Estudiantil para el Fortalecimiento de la Convivencia Escolar.

Ministerio de Educación [MINED]. (2008b). Normativa de funcionamiento. Documento 5, Gestión Escolar Efectiva. https:/www.transparencia.gob.sv/institutions/ mined/documents/104139/download

Ministerio de Educación [MINED]. (2018d). Caja de herramientas para docentes y educadores "Ciudadanía, Memoria y Cultura de Paz en El Salvador. Convivencia y cultura de paz" (Vol. 3). 192.168.8.1/html/guide. html? randid $=1858932887$ ? updataredirec$\mathrm{t}=$ www.auschwi tzinstitute.org/es/ciudadania-memoria-y-cultura-de-paz-en-el-salvador-caja-deherramientas-para-docentes-y-educadores/ 
Monsalud Gallardo, G. (2016). La Escuela de contexto rural ¿De la diferencia a la desigualdad? Revista Iberoamericana de Educación, 55(5), 1-10. https://rieoei.org/historico/deloslectores/3919Gallardo.pdf

Murguialday, C. y Vásquez, N. (2005). Un paso más: evaluación del impacto de género. Cooperacció. http://www.mujeresenred.net/ IMG/pdf/Un_paso_mas_evaluacion_del_ impacto_de_genero.pdf

Ordóñez-Matamoros, G. (Dir.). (2013). Manual de análisis y diseño de políticas públicas. Universidad Externado de Colombia.

Romero, R. (2005). Democracia participativa, una utopía en marcha reflexiones, experiencias y un análisis del caso porteño. Ediciones Cooperativas.

Sabucedo, J. M. (1988). Participación política. En J. Seoane y A. Rodríguez, Psicología política (pp. 165-194). Pirámide.

Santos Guerra, M. Á. (Coord.). (2003). Aprender a convivir en la escuela. Akal.

Sarramona i López, J. y Rodríguez Neira, T. (2010). Participación y calidad de la educación. Aula Abierta,
38(1), 3-14. https://digibuo.uniovi.es/ dspace/bitstream/handle/10651/10583/ AulaAbierta.2010.38.1.3-14.pdf;jsessioni$\mathrm{d}=$ A8461DF6F8B1137663F9E902323DC6E$\mathrm{F}$ ?sequence $=1$

Seca, M. V. (2016). El lugar del género en los estudios de participación juvenil [Ponencia]. I Jornadas Nacionales de Investigación en Ciencias Sociales de la UNCuyo "Perspectivas actuales en la investigación en ciencias sociales: problemáticas, enfoques epistemológicos y abordajes teórico-metodológicos". https://www.academia. edu/38355407/El_lugar_del_g\%C3\%A9nero_ en_los_estudios_de_participaci\%C3\%B3n juvenil

Instituto Nacional de las Mujeres [INMUJERES]. (2007). Glosario de género. http://cedoc.inmujeres.gob.mx/documentos download/100904.pdf

Vásquez Recio, R. M. (2016). Las escuelas públicas rurales: entre el bien común y la exclusión. Revista Interuniversitaria de Formación del Profesorado, 85, 67-79. https://dialnet.unirioja.es/servlet/ articulo? codigo $=5573946$ 